\title{
2.7 Intermedialität: Text/Bild-Verhältnisse
}

\author{
Gabriele Rippl
}

\section{Einleitung}

In der Literatur-, Kultur- und Theaterwissenschaft, der Kunstgeschichte, der Bildund Filmwissenschaft ist Intermedialität in den letzten Jahrzehnten zu einem zentralen theoretischen Konzept geworden. Allgemein gesprochen bezeichnet der Begriff ,Intermedialität' Beziehungen zwischen verschiedenen Medien, beispielsweise die zwischen Texten und (statischen und beweglichen, analogen und digitalen) Bildern oder zwischen Texten und Musik. Der Erfolg der Intermedialitätsforschung hat mehrere Gründe, einer der wichtigsten ist die intermediale Qualität zahlreicher Artefakte und kultureller Phänomene der Gegenwartskultur, welche die Grenzen ihres eigenen Mediums thematisieren und auf vielfältige und innovative Weise überschreiten. Produktionen wie The Fall of the House of Usherettes von Forkbeard Fantasy (1996) oder Gob Squad's Kitchen von Gob Squad (1994; vgl. Georgi 2014) sind nur zwei Beispiele für die intermediale Experimentierfreudigkeit des Gegenwartstheaters und der Performance Art. Auch bei der ins Internet eingestellten Performance-Poesie beziehungsweise Spoken-WordDichtung, die an mittelalterliche Liedvorträge erinnert und meist die Länge von Popsongs hat, handelt es sich um komplexe intermediale Konstellationen der Gegenwartskultur, deren Beliebtheit sich an den zahlreichen Klicks ablesen lässt. Beispiele sind die über YouTube zugänglichen poetry clips von Bas Böttcher (etwa „Dot-Matrix“), Fabian Navarro („Schlaflos“), Jürg Halter („,Spiegelbild“) sowie Maximilian Humpert und Svenja Gräfen („Stillstand“).

Wendet man sich der (anglofonen) Gegenwartsliteratur zu, dann zeigen sich ebenfalls ausgeprägte intermediale Verfahren: Romane, Erzählungen und Gedichte enthalten häufig Abbildungen und Diagramme, sie diskutieren abwesende Bilder oder evozieren mit verbalen Mitteln Bilder und Bildverfahren. In der britischen und irischen Gegenwartsliteratur setzen sich unter anderem A. S. Byatt (The Matisse Stories, 1993, und Still Life, 1985), John Banville (The Book of Evidence, 1989) und Adam Thorpe (Ulverton, 1992) mit Bildern auseinander; in den postkolonialen Literaturen sind etwa Autorinnen und Autoren wie David Dabydeen (Turner, 1994), Salman Rushdie (The Moor's Last Sigh, 1995) oder Raj Kamal Jha (Fireproof, 2006) und in der nordamerikanischen Literatur Michael Ondaatje (Running in the Family, 1982), Charles Simic (Dime-Store Alchemy, 1992), Carol Shields (The Stone Diaries, 1993), Mark Z. Danielewski (House of Leaves, 2000), John Updike (Seek My Face, 2002), Siri Hustvedt (What I Loved, 2003) oder Jona- 
than Safran Foer (Extremely Loud and Incredibly Close, 2005) für ihre intermedialen Grenzgänge bekannt. $\mathrm{Zu}$ den erfolgreichsten intermedialen, Text und Bild kombinierenden Genres der Gegenwartskultur gehört nicht zuletzt die grafische Literatur (Comics, graphic novels etc.; siehe 4.15 BöGER).

Intermedialität ist ein theoretisches Konzept, welches erlaubt, literarische Texte mit Blick auf ihre Rolle in medialen Netzwerken in historischer und systematischer Perspektive zu analysieren (vgl. Rippl 2005a). Die Intermedialitätsforschung ermöglicht folglich die Untersuchung von komplexen Mechanismen der Bedeutungsbildung in und durch inter- und multimediale Konstellationen und stellt dabei das Erklärungspotential von Sprachmodellen für andere Medien wie Bilder oder Musik infrage. Zudem privilegiert sie ein ,demokratisches' Vorgehen, das heißt, sie beschäftigt sich nicht ausschließlich mit hochkulturellen Kunstformen, sondern auch und gerade mit Produkten der Populärkultur und den neuen Medien. Literatur, Zeitungen, Oper, Popmusik, Malerei, Film, Comics und Videokunst sind gleichberechtigte mediale Phänomene, deren Allianzen und Konkurrenzen die Intermedialitätsforschung in ihren jeweiligen kulturhistorischen Konstellationen analysiert. Dabei legte sie zunächst den Fokus auf Medienunterschiede, untersucht heute jedoch zunehmend mediale Überlappungen und Netzwerke sowie deren kulturelle Funktionen.

\section{Text/Bild-Konzeptualisierungen: Ein historischer Überblick}

Die literaturwissenschaftlichen Debatten um W. J.T. Mitchells Buch Bildtheorie (1994; Picture Theory) und den darin proklamierten pictorial turn haben wesentlich dazu beigetragen, dass literarische Texte heute vermehrt als Teil einer visual culture auf ihre Kollaborationen und Kompetitionen mit visuellen Medien befragt werden. Die Intermedialitätsforschung - und im Besonderen die Erforschung von Text-Bild-Beziehungen - setzt jedoch keineswegs erst im späten 20. Jahrhundert ein, sondern beginnt in der Antike. Seither wurde das Verhältnis von Text und Bild, von Literatur und visuellen Medien von Epoche zu Epoche sowie innerhalb einzelner Epochen sehr unterschiedlich konzeptualisiert. Zunächst wurde in der griechisch-römischen Antike die strukturelle Ähnlichkeit von Text und Bild und damit ihr Analogieverhältnis betont. Horaz hielt in seiner Ars poetica (ca. $14 \mathrm{v}$. Chr.) die Simonides von Keos (spätes 6. Jh. v. Chr.) zugeschriebene einflussreiche ut pictura poesis-Formel fest, die folgendermaßen ausgelegt wurde: Malerei sei ,stumme Dichtung‘ und Dichtung ,redende Malerei‘ (vgl. 3.1 WETZEL). Noch in der Frühen Neuzeit lässt sich der Einfluss dieser Formel ausmachen. Allerdings verdeckt die nun gängige Redeweise von den komplementären ,Schwesterkünsten` 
(vgl. Hagstrum 1958), dass sich Dichtung, Malerei und Bildhauerei zunehmend als konkurrierende Kunstformen verstanden, denn Maler wie Leonardo da Vinci haben die bildende Kunst aufgrund ihrer besseren Erfüllung des Ähnlichkeitspostulats aus dem Stand eines dienenden Handwerks in den Rang einer eigenständigen, der Dichtung überlegenen Kunst gehoben. Ein auffälliges Charakteristikum der frühneuzeitlichen Kultur war folglich der Wettkampf zwischen den Künsten, der sogenannte paragone (vgl. u. a. Preimesberger 2011). In England setzten sich zum Beispiel Dichter wie Edmund Spenser, Philip Sidney und William Shakespeare in ihren Werken mit den visuellen Medien der Zeit (Malerei, Zeichnung, Druckgrafik, Bildhauerei und Gobelinkunst) auseinander (vgl. Rippl 2005b). An ihren Texten lässt sich die neue, vom Neoplatonismus eingeleitete Wertschätzung des Visuellen und die Orientierung an der in Quintilians antiker Rhetorik Institutio oratoria (95 n. Chr.; Ausbildung des Redners) propagierten sprachlichen Qualität der Anschaulichkeit (griech. enargeia, lat. evidentia) ablesen (siehe 2.2 BERNDT).

Im 18. Jahrhundert verabschiedete Gotthold Ephraim Lessing das mediale Analogieverhältnis und arbeitete in seiner wirkungsmächtigen Schrift Laokoon oder Über die Grenzen der Malerei und Poesie (1766) grundlegende mediensemiotische Unterschiede zwischen Dichtung und Malerei, Text und Bild heraus (vgl. 2.3 SCHNEIDER): Während die Dichtung dem Prinzip der Sukzessivität unterliege und eine Zeitkunst sei, folge die Malerei den Gesetzen der Simultaneität und gehöre zu den Raumkünsten. Es gibt jedoch gute Gründe, die von Lessing vorgeschlagene strikte kategoriale Trennung der Künste zu hinterfragen, denn diese weisen semiotisch und medial gesprochen nicht nur Unterschiede, sondern auch Gemeinsamkeiten auf - zum Beispiel hinsichtlich der Rezeption. Deshalb überrascht es nicht, dass die Romantikerinnen und Romantiker nur wenige Dekaden später nach dem Gesamtkunstwerk strebten, das Lessings Trennungsversuch der Künste/Medien durch die Vorstellung ihrer Verwandtschaft wieder nivellierte.

Im 20. Jahrhundert etablierten sich dann in der Literaturwissenschaft die sogenannten interart studies beziehungsweise comparative arts, die sich dem Vergleich der Künste widmeten und erneut Analogien zwischen Bildkunst und Literatur hervorhoben (vgl. Weisstein 1992). Dagegen betonte die Philosophin Susanne K. Langer aus der Perspektive der „symbolischen Logik“ die Unterschiede zwischen Wörtern und Bildern (Gemälden, Zeichnungen und Fotografien): „Wörter kennen nur eine lineare, gesonderte, sukzessive Ordnung; sie reihen sich, wie die Perlen eines Rosenkranzes, eins ans andere; [...] es [gibt] für uns keine Möglichkeit, in simultanen ,Namensbündeln' zu sprechen. Wir müssen ein Ding nach dem anderen beim Namen nennen“ (Langer 1984 [1942], 87). Die sequentielle Qualität von Sprache wird laut Langer dann zum Problem, wenn man in Beschreibungen dazu gezwungen ist, die simultane Gegenwärtigkeit der Dinge, ihren prä- 
sentativen Charakter, in Form von nacheinander aufgereihten Ideen darzubieten, und das, „obgleich Gegenstände ineinanderliegen; so wie Kleidungsstücke, die übereinander getragen werden, auf der Wäscheleine nebeneinander hängen“ (ebd., 88). Dinge, die sich der diskursiven Darstellung nicht fügen - so folgert Langer -, können eigentlich nicht zur Sprache gebracht werden. Visuelle Formen wie Linien, Farben, Proportionen usw. sind ebenso „der Artikulation, d. h. der komplexen Kombination fähig wie Wörter. Aber die Gesetze, die diese Art von Artikulation regieren, sind von denen der Syntax, die die Sprache regieren, grundverschieden. Der radikalste Unterschied ist der, daß visuelle Formen nicht diskursiv sind“ (ebd., 99). An Langers Konzeptualisierung der Unterschiede zwischen Text und Bild lässt sich das Nachwirken der ,Laokoon-Debatte“ ablesen, das bis heute andauert und entscheidende Impulse für die Weiterentwicklung einer postklassischen, intermedialen Narratologie geliefert hat (vgl. Wolf 2002).

\section{Intramedialität - Intermedialität - Transmedialität: Definitionen und Typologien}

Der historische Überblick hat verdeutlicht, dass das Verhältnis von Text und Bild sehr unterschiedlich konzeptualisiert wurde. Der substantialisierenden Festschreibung von Medienunterschieden einerseits stehen die Betonung der Gemeinsamkeiten von Text und Bild und die Ablehnung einer strikten Trennung andererseits gegenüber. Diese divergierenden Perspektiven auf mediale Differenzen beruhen nicht zuletzt auf der Semantik des Medienbegriffs selbst: ,Medium“ heißt wörtlich so viel wie ,Mitte` oder ,Vermittler` und ist erst spät, am Ende des 19. Jahrhunderts, im Zusammenhang mit dem Spiritismus in Lexika nachgewiesen (vgl. Faulstich 1991, 8; Rippl 2012). Medien beziehen sich auf ein ,Zwischen“, da sie Informationen kodieren und die Distanz zwischen Sender und Empfänger überbrücken, wobei im Sinne des berühmten Diktums von Marshall McLuhan „the medium is the message“ davon auszugehen ist, dass das jeweilige Medium die Botschaft immer auch mitformt. Auch Marie-Laure Ryan geht davon aus, dass Medien keineswegs als bloße Übertragungskanäle zu verstehen sind: „[They] are not hollow conduits for the transmission of messages but material supports of information whose materiality, precisely, ,matters' for the type of meanings that can be encoded“ (Ryan 2004, 1-2). Ryan ordnet die zahlreichen Definitionsangebote in drei (sich ergänzende) Kategorien ein: (1.) semiotische Definitionen des Mediums, die sich auf unterschiedliche Codes und die jeweils adressierten Sinneskanäle beziehen und drei übergeordnete Medientypen - verbale, visuelle und auditive - unterscheiden; (2.) material-technologische Ansätze, welche die 
Materialien und Technologien analysieren, auf die sich semiotische Typen von Medien stützen; sowie (3.) kulturelle Definitionen, die an sozialen und kulturellen Aspekten von Medien und ihrer Rolle im Mediennetzwerk interessiert sind (vgl. ebd., 14-16).

Lange diente ,Intertextualität‘ als Oberbegriff auch für intermediale Phänomene. Heute werden intertextuelle Verweise, also Text-Text-Beziehungen, gelegentlich unter dem Stichwort ,Intramedialität‘ verhandelt, weil die Beziehungen ,innerhalb eines Mediums bestehen, mit denen also eine Überschreitung von Mediengrenzen nicht einhergeht“ (Rajewsky 2004, 12). Von Intramedialität unterschieden wird ,Intermedialität', ein Begriff, der wörtlich ,zwischen den Medien' bedeutet und damit die Semantik des ihm zugrunde liegenden Begriffs Medium (der sich ja bereits auf ein ,Zwischen“ bezieht) noch einmal verdoppelt. Der Begriff ,Intermedialität‘ wurde 1983 durch Aage A. Hansen-Löve im Zusammenhang mit den ,intermediale[n] Beziehung[en] zwischen Gattungen (bzw. Einzeltexten) verschiedener Kunstformen“ (Hansen-Löve 1983, 291) in die literaturwissenschaftliche Diskussion eingeführt, jedoch prägte Dick Higgins bereits 1966 den verwandten Begriff intermedia (Higgins 1984; vgl. zur Entwicklung des Konzepts ,Intermedialität‘ Todorow 2011 sowie Paech und Schröter 2008). Einflussreiche Definitionen stammen von Werner Wolf (Wolf 2005) und Irina Rajewsky; letztere bestimmt Intermedialität als ein „Hyperonym für die Gesamtheit aller Mediengrenzen überschreitenden Phänomene“ (Rajewsky 2004, 14), welches mindestens „,zwei konventionell als distinkt wahrgenommene Medien“ involviert (ebd., 13).

Rajewsky zufolge dreht sich die derzeitige Debatte um zwei Auffassungen von Intermedialität: ,a broader and a narrower one, which are not in themselves homogeneous. The first concentrates on intermediality as a fundamental condition or category while the second approaches intermediality as a critical category for the concrete analysis of specific individual media products or configurations“ (Rajewsky 2005, 47). Rajewskys literarische Konzeption von Intermedialität im letzteren, engeren Sinn umfasst drei Kategorien und geht von klaren Mediengrenzen aus. Wie die Diskussion einiger Beispiele zeigen wird, weisen mediale Konfigurationen natürlich häufig Merkmale auf, die sich mehr als nur einer dieser drei Kategorien zuordnen lassen: (1.) Medienkombination, das heißt ein „mediales Zusammenspiel, Medien-Fusion, Multi-, Pluri- oder Polymedialität“ (Rajewsky 2004, 14), Beispiele sind Oper, Film, Theater, Comics, illustrierte Romane etc.; (2.) „Medienwechsel“ oder „Medientransfer“ beziehungsweise „Medientransformation“, das heißt „Literaturverfilmungen bzw. Adaptionen, ,Veroperungen“, ,Buch zum Film“ etc.“ (ebd., 15); (3.) „,intermediale Bezüge“, das heißt „der Bezug eines literarischen Textes, eines Films oder Gemäldes auf ein bestimmtes Produkt eines anderen Mediums oder auf das andere Medium“ (ebd., 16). Beispiele sind die 
Musikalisierung von Erzählliteratur, visuelle und filmische Schreibmodi in der Literatur (zoom shots, montage editing) und am Visuellen orientierte Beschreibungsmodi in der Literatur, zum Beispiel Bildbeschreibungen (Ekphrasen).

W. J.T. Mitchell hat bemerkt, „all media are mixed media“ (Mitchell 1994, 94-95). Gerade weil Medienfusionen und Medientranspositionen ein so zentrales Merkmal der Gegenwartskultur sind, wurde in jüngster Zeit vermehrt die Frage gestellt, ob es denn überhaupt sinnvoll ist, Einzelmedien separat zu untersuchen und von kategorialen Mediengrenzen auszugehen. Infolge dieser Diskussion wurde auch das Konzept der Intermedialität kritisch beleuchtet, weil es die Mediengrenzen, deren Überschreitung es behauptet, gleichzeitig immer auch voraussetzt und bestätigt (vgl. Weingart 2010). Die Vorstellung rigider, substantieller Mediengrenzen geriet unter Essentialismusverdacht, und Text-BildUnterscheidungen wurden in der Folge als relationale Konstellation aufgefasst, deren Bestandteile zwar situativ zu bestimmen, nicht aber zu substantialisieren sind. Dennoch erscheint es Forscherinnen und Forschern wie Marie-Laure Ryan (2005), Irina Rajewsky (2010) und Werner Wolf (2011) problematisch, das Konzept der Mediengrenze gänzlich aufzugeben: „Currently, efforts are being made to strengthen common and crossover features [...] in intermediality studies [...]. Contrary to this tendency, I have advanced the thesis that medial differences and the notion of media borders play a crucial and extremely productive role in the context of intermedial practices. [...] [I]t is precisely the concept of the border which can be strengthened." (Rajewsky 2010, 63-64) Andere Forscherinnen und Forscher, etwa Brigitte Weingart, betonen mit Berufung auf W. J. T. Mitchell, Jacques Derrida und andere viel stärker die Durchlässigkeit zwischen den Medien und damit auch der Text-Bild-Unterscheidung (vgl. Weingart 2001) und warnen mit Blick auf vermeintliche Mediengrenzen vor Essentialismen: „In Abgrenzung auch zu einem Medienpurismus [...] gehen wir davon aus, dass die mediale Differenz von Bild und Text jeweils nur als Grenzziehung thematisch und beobachtbar wird. Das bedeutet zugleich, dass sich das Verhältnis von Bild und Text nur als Differenz in einer konkreten Situierung dieser Grenze beschreiben lässt und dass sich Aussagen zum Status des Bildes und des Textes nur in einem historisch-kulturellen Kontext machen lassen, der es überhaupt erst ermöglicht, die Unterscheidung von Bildern und Texten als solche still zu stellen." (Voßkamp und Weingart 2005, 10) Gerade weil intermediale Artefakte und Phänomene Mediengrenzen durchlässig werden lassen, gilt es, starre, essentialistisch gedachte Mediengrenzen zu vermeiden und jedweden Medienpurismus zu hinterfragen (siehe dazu auch 3.6 BENTHIEN, 4.14 WEINGART).

In diesem Zusammenhang ist das Konzept der Transmedialität von Interesse, anhand dessen „medienunspezifische Phänomene, die in verschiedensten Medien mit den dem jeweiligen Medium eigenen Mitteln ausgetragen werden 
können, ohne daß hierbei die Annahme eines [...] Ursprungsmediums wichtig oder möglich ist“ (Rajewsky 2002,13). Das Konzept der Transmedialität ist jüngst insbesondere im Forschungsgebiet der postklassischen Narratologie, das sich unter anderem mit dem Narrationspotential von Bildern und Musik beschäftigt, gewinnbringend weiterentwickelt und operationalisiert worden (vgl. Ryan 2005; Wolf 2011). Für Marie-Laure Ryan ist Transmedialität dann gegeben, wenn eine Geschichte, die zunächst in einem Medium erzählt wird, später in einem anderen Medium erneut erzählt wird; allerdings wird das Ergebnis aufgrund von Medienspezifika nie dasselbe sein (Ryan 2004, 31-32). Als Beispiele für transmediale Phänomene und Prozesse der Gegenwartskultur lassen sich gemäß Ryan Comics und graphic novels anführen, die durch die charakteristischen Text-Bild-Kombinationen nicht nur intermedial verfasst sind, sondern von denen einige zudem erfolgreich verfilmt wurden. Ein Beispiel ist James McTeigues Filmversion (2005) von Alan Moores und David Lloyds V for Vendetta (1982-1988), das zudem 2006 von Stephen Moore als Roman adaptiert wurde. Ein weiteres Beispiel für Transmedialität ist Paul Karasiks und David Mazzucchellis gleichnamige Adaption von Paul Austers postmodernem Roman City of Glass (1985) im graphic novel-Format (2004). Ein weiteres treffendes Beispiel für Adaptionsprozesse ist das Multimediaprojekt Die Dichte (2012) der französischen Schriftstellerin Marie NDiaye, dem ein Langgedicht NDiayes zugrunde liegt, welches in verschiedenen Gattungen und Medien adaptiert und aufgeführt wurde, als live performance, als Kunstfilm und als Hörspiel im Radio.

\section{Zur Intermedialität von Text und Bild: Literarische Beispiele}

Typologien intermedialer Phänomene laufen Gefahr, Mediengrenzen und -unterschiede allererst an Artefakten hervorzutreiben, die gerade darauf angelegt sind, Mediengrenzen zu überwinden. Dennoch ist es heuristisch sinnvoll, Text-BildBeziehungen in der Literatur nach Gruppen zu unterteilen, auch wenn viele literarische Beispiele mehr als nur einer Gruppe zuzuordnen sind. Es lassen sich unterscheiden: a) Schrift-Bilder produzierende typografische Experimente, b) Texte, die mit Abbildungen, also konkreten Bildern versehen sind, und c) Texte, die intermediale Bezüge herstellen, indem sie das Bildmedium verbal evozieren. 


\section{Schrift-Bilder: Typografische Experimente}

Im Rahmen der bis ins 18. Jahrhundert verbindlichen imitatio-Lehre und MimesisÄsthetik versuchten Dichter wie Edmund Spenser und Philip Sidney möglichst lebendige, anschauliche verbale Bilder zu schaffen. In diese literarische Tradition gehören auch die in der Frühen Neuzeit außerordentlich beliebten Figurengedichte (vgl. Adler und Ernst 1987). Bei Figurengedichten, auch ,Technopägnien` genannt, ergibt die Anordnung der Buchstaben und Wörter eine ikonische Form. Ein Beispiel für ein solches intermediales Verfahren liefert der metaphysical poet George Herbert mit seinem Gedicht „Easter-wings“ (Abb.1; vgl. Rippl 2005a, 40-41).

GEORGE HERBERT

Easter-mings

Lord, who createdst man in wealth and store,

Though foolishly he lost the same,

Decaying more and more,

Till he became

Most poore:

With thee

$\mathrm{O}$ let me rise

As larks, harmoniously,

And sing this day thy victories:

Then shall the fall further the flight in me.

My tender age in sorrow did beginne:

And still with sicknesses and shame

Thou didst so punish sinne,

That I became

Most thinne.

With thee

Let me combine

And feel this day thy victorie:

For, if I imp my wing on thine, Affliction shall advance the flight in me.
Abb. 1: George Herbert: Easter-wings, Figurengedicht, 1633

Dieses Figurengedicht, das den Aufstieg der menschlichen Seele zu Gott beschreibt, kann durch die für Texte übliche lineare Rezeptionsweise nicht gänzlich erfasst, sondern muss auch als Bild zweier Stundengläser - als Leitsymbol der Vergänglichkeit des Lebens - beziehungsweise (dreht man den Text um neunzig Grad) einer doppelten Flügelform gesehen werden. Die Diskursivität von Schrift ist hier eng mit Präsentativität, das heißt ihrem visuellen Aspekt verknüpft, was die Semantik des Textes durch seine ikonische Gestaltung verdichtet. Figurengedichte erfreuten sich bereits in der Antike großer Beliebtheit, aber auch noch in der Moderne (etwa in den historischen Avantgarden wie dem Dadais- 
mus und der Konkreten Poesie) und Postmoderne werden sie von zahlreichen Autoren geschätzt. In dem Figurengedicht „Swan and Shadow“ (1969) des amerikanischen Dichters John Hollander zum Beispiel, wird das Thema, ein Schwan und sein Schatten, nicht nur beschrieben, sondern anhand der ikonischen Anordnung der (auf einer Schreibmaschine getippten) Buchstaben auch visuell präsentiert. Die Inhaltsebene des Gedichts - die philosophischen Reflektionen über den Schwan und seinen Schatten - wird also eng mit der Präsentationsform verbunden. Lauren Redniss' experimentelle grafische Biografie der Atomphysiker Marie und Pierre Curie, Radioactive - Marie \& Pierre Curie. A Tale of Love and Fallout (2010), lässt sich ebenfalls als ein Beispiel für die anhaltende Relevanz technopägnischer Verfahren in der grafischen Gegenwartsliteratur anführen (vgl. Redniss 2010, 124-125; Abb. 2). Auch bei diesem Beispiel werden die dargestellte Person und ihre Geschichte nicht nur beschrieben, sondern die Wörter auf der Seite werden so angeordnet, dass sie die Form des Kopfes und Oberkörpers der Person wiedergeben.

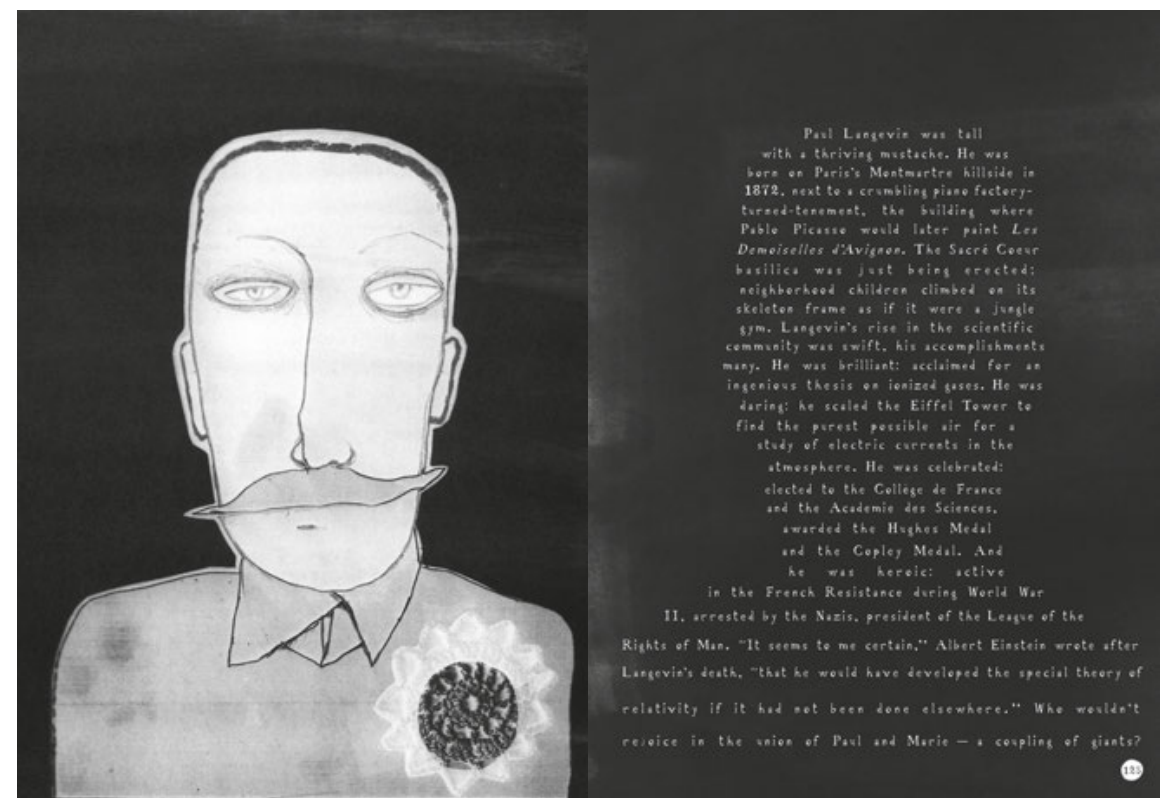

Abb. 2: Lauren Redniss: Radioactive - Marie \& Pierre Curie. A Tale of Love and Fallout, 2010 


\section{Kopräsenz von Text und konkretem Bild}

Comics und graphic novels - etwa Neil Gaimans zehn-bändige Sandman-Serie (1989-1996), Alan Moores Watchmen (1986-87) und Art Spiegelmans Maus (1980-1991) - gehören heute zu den populärsten Beispielen komplexer Text-BildKombinationen, die dieser zweiten Gruppe zuzuordnen sind (siehe 4.15 BöGER). In graphic novels kommt Bildern eine tragende Rolle $\mathrm{zu}$, sie werden dem Text kaum untergeordnet. Anders verhält es sich bei den insbesondere in der Frühen Neuzeit weit verbreiteten Emblemen (vgl. Henkel und Schöne 1996 [1967]), welche ebenfalls Text und konkrete Bilder kombinieren (siehe 4.4 NEUBER). Das bimediale Emblem weist eine dreiteilige Struktur auf: ein schriftliches Motto inscriptio beziehungsweise ,Inschrift‘), eine pictura (,Bild`) und eine subscriptio (,Unterschrift', das heißt ein Text in Form eines Gedichtes). Diese subscriptio schränkt das - häufig enigmatische - Bild in seiner Polysemie ein und legt es auf eine bestimmte Bedeutung fest.

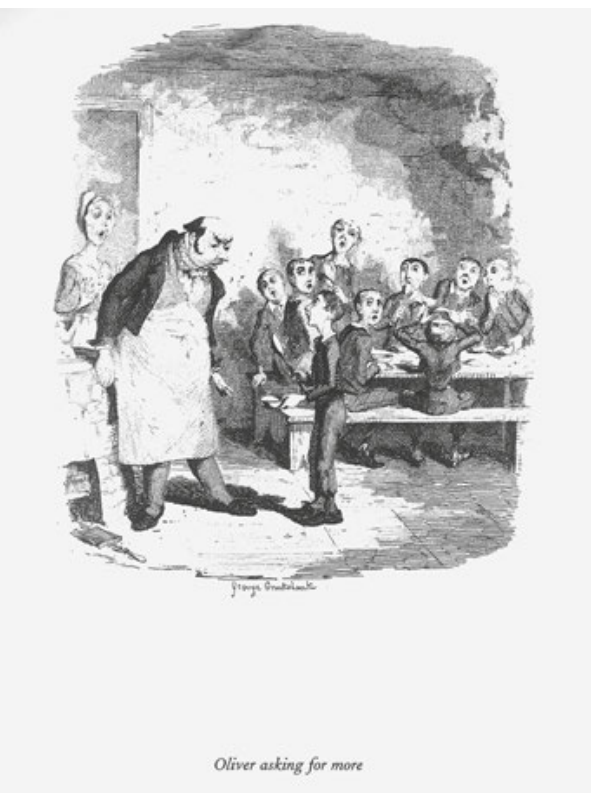

Abb. 3: Illustration/Frontispiz aus der Erstausgabe von Charles Dickens' Oliver Twist, 1838, Illustrator: George Cruikshank

Als weitere Kategorie in der Gruppe der Text-Bild-Kombinationen sind die schon seit dem Mittelalter verbreiteten illustrierten Texte zu nennen. So lenken etwa die den Romanen von Charles Dickens beigegebenen Bilder die Aufmerksamkeit der Leserinnen und Leser, indem sie die Semantik wichtiger Textpassagen visuell verdichten - wie das Bild des schmächtigen Oliver Twist verdeutlicht, der um mehr 
Suppe bittet (Abb. 3) und das die missliche Lage der hungernden Waisenkinder ebenso eindrücklich vor Augen führt wie die unerbittliche, korrupte Natur der Wärter. Demgegenüber hat die Illustration heute längst ihre dienende Rolle als Erleuchtung und Veranschaulichung des Textes abgelegt und ein mit dem Text konkurrierendes Eigenleben entwickelt. So finden sich in Foers Roman Extremely Loud and Incredibly Close (2005) viele Bilder, bei denen es sich allerdings selten um Illustrationen im traditionellen, oben genannten Sinn handelt; vielmehr sind die Text-Bild-Beziehungen meist enigmatisch und laden zum Rätseln und Spekulieren ein (Abb. 4; was das Türschloss mit dem Satz auf der gegenüberliegenden Seite zu tun hat, bleibt unklar). Dies trifft auch auf einige der Text-Bild-Interaktionen in W. G. Sebalds Roman Austerlitz (2001) und seinem Reisebericht Die Ringe des Saturn (1995) zu. In den letzten Jahren wurden zahlreiche Romane publiziert, denen die Autorinnen und Autoren Bilder in Form von Fotografien beigegeben haben: Raj Kamal Jhas Fireproof (2006) und Aleksandar Hemons The Lazarus Project (2008) sind zwei Beispiele, in denen, wie bei Foer, die im 19. Jahrhundert noch typische Illustrationsfunktion von Bildern häufig außer Kraft gesetzt und die für die Fotografie typische Funktion der Authentifizierung des Dargestellten ironisch unterlaufen wird.

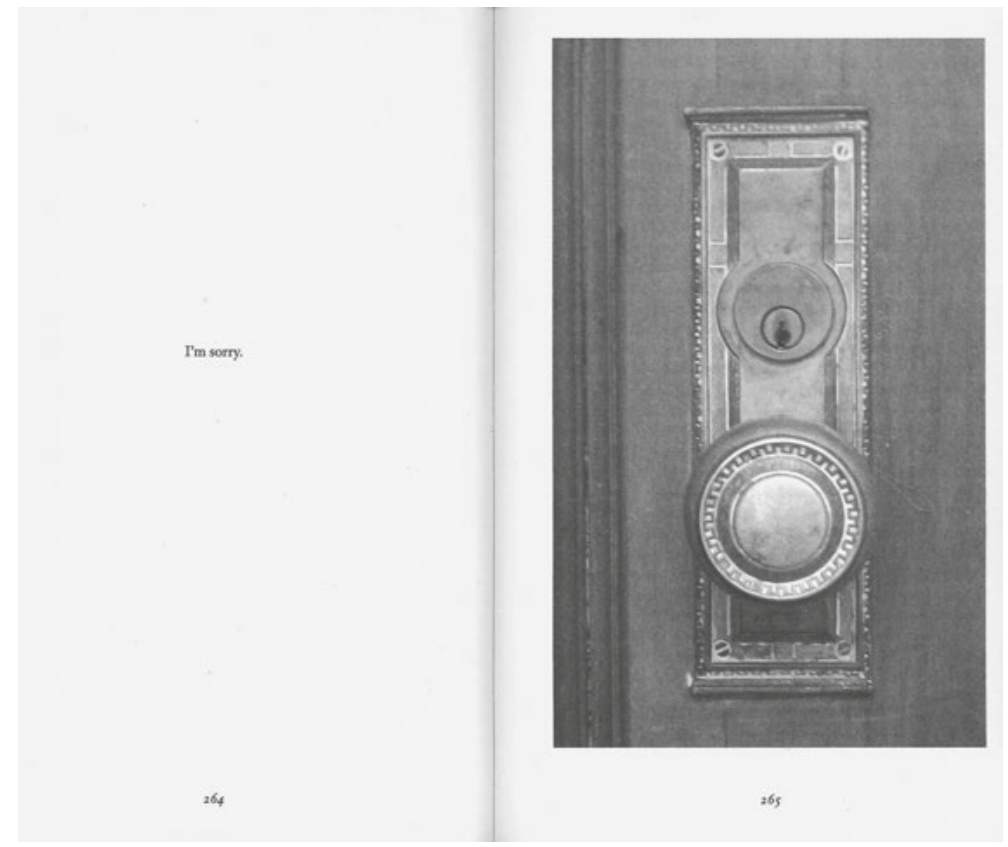

Abb. 4: Buchseiten aus Jonathan Safran Foer: Extremely Loud and Incredibly Close, 2005 
Diese Beispiele legen nahe, dass Kategorisierungen immer nur erste Annäherungsversuche an die komplexen, multifunktionalen intermedialen Experimente sein können. So wurden zum Beispiel Embleme in der zweiten Gruppe von TextBild-Kombinationen gelistet; wenn Embleme jedoch in den Textteilen Beschreibungsstrategien einsetzen, die ihrerseits Visualität produzieren, dann können sie zusätzlich in der dritten Gruppe verortet werden.

\section{Intermediale Bezüge: Pikturalismus - Ekphrasis}

Texte können mithilfe spezifischer Beschreibungsstrategien Bilder evozieren, die als solche jedoch nicht medial in Erscheinung treten. Häufig werden bei der Untersuchung solcher intermedialer Verfahren, bei denen auf Bilder lediglich verbal verwiesen wird, pikturalistische Beschreibungen von ekphrastischen unterschieden: Wenn mittels sprachlicher Beschreibung Bildeffekte erzielt und Bildqualitäten evoziert werden, indem man Personen, Landschaften und Objekte so beschreibt, als handelte es sich um tatsächlich vorhandene oder fiktive Kunstwerke, dann spricht man von Pikturalismus (vgl. Bender 1972; Torgovnick 1985). Der amerikanische Lyriker William Carlos Williams hat beispielsweise in seinem berühmten modernistischen Gedicht „The Red Wheelbarrow“ (1923) pikturalistische Strategien eingesetzt, die das, was sie beschreiben, so präsentieren, als handle es sich dabei um ein konkretes, materiales Bild (was auch an dem verbalen Rahmungsverfahren in den Zeilen 1-2, „so much depends/upon“, abzulesen ist, das der Aufmerksamkeitslenkung dient und den Bildeffekt verstärkt).

The Red Wheelbarrow

so much depends

upon

a red wheel

barrow

glazed with rain

water

beside the white

chickens.

(Williams 1991 [1923], 224) 
Ende der 1980er Jahre wurde die Ekphrasis, das heißt die Bildbeschreibung, zum Gegenstand literaturtheoretischer Debatten (vgl. Krieger 1992; Heffernan 1991 und 1993; Boehm und Pfotenhauer 1995; Hollander 1995; Mitchell 1990 und 1994; Wagner 1996; Klarer 1999; Robillard und Jongeneel 1998; Schmitz-Emans 1999; Wandhoff 2003; Rippl 2005a) und ist heute - trotz anspruchsvoller interdisziplinärer methodologischer Herausforderungen - zu einem wichtigen Forschungsgebiet der Literatur-, Kultur- und Kunstwissenschaft geworden (siehe 4.1 WANDHOFF). Der Begriff stammt aus dem Griechischen, setzt sich aus ,ek' (,aus`) und ,phrazein' (,zeigen', ,bekannt, deutlich machen') zusammen und bedeutet so viel wie ,völlig, restlos deutlich machen beziehungsweise zeigen'. Gemäß der Definition von James A. W. Heffernan steht Ekphrasis für „the verbal representation of visual representation“, das heißt für die Beschreibung von real existierenden oder fiktiven Werken bildender Kunst (Heffernan 1993, 2). Heffernans Definition, die eine Ekphrase als eine Repräsentation zweiten Grades bestimmt, hat den Vorteil, dass sie die reflexive Ebene betont, die Ekphrasen auszeichnet: Sie reflektieren immer das eigene wie das fremde ,Repräsentationsmedium‘ (also die Bilder) mit und tragen damit wesentlich zur Problematisierung von Mimesis-, Referentialitäts- und Repräsentationskonzepten bei. Hingegen ergibt sich der Nachteil von Heffernans Ansatz aus dem Repräsentationsbegriff selbst, der im Verdacht steht, eine Allianz mit Konzepten wie Referentialität und Ähnlichkeit zu bilden.

Ein ekphrastisches Gedicht oder eine ekphrastische Passage in einem Prosatext kann visuelle Objekte und Qualitäten sehr unterschiedlich evozieren: von kürzeren, abbreviatorischen Verfahren bis hin zu äußerst detail- und umfangreichen Ekphrasen ist alles möglich. Während ekphrastische Gedichte das Interesse der Forschung besonders oft auf sich gezogen haben, wurde die Form der narrativen Ekphrasis lange vernachlässigt. Folgt man Tamar Yacobi, dann ist dafür die Tendenz vieler Theoretikerinnen und Theoretiker verantwortlich, Text-BildRelationen immer noch anhand von Mimesis-Modellen und irreführenden, vermeintlichen Dichotomien (episch vs. lyrisch; Handlung vs. Beschreibung; Narrativität vs. Pikturalität) bestimmen zu wollen (Yacobi 1995, 600-602). Während die klassische Narratologie die Deskription noch als narrative Pause verstand, die die Präsentation der Handlungskette unterbricht, so machen neuere narratologische Untersuchungen wie die von Ruth Ronen (1997) deutlich, dass die Beschreibung mit Blick auf die Erzählung keine ausschließlich dienende, amplifikatorische Rolle einnimmt und auch nicht als Gegenbegriff zur Erzählung verstanden werden muss. Um zu verdeutlichen, dass von Beschreibungen auch narrative Impulse ausgehen und Erzählelemente der Beschreibung dienen können, hat Harold Mosher die Begriffe „narratized descriptions“ und „descriptized narrations“ (Mosher 1991, 426) in die Diskussion eingebracht, welche verdeutlichen, dass ,reine Beschreibung‘ und ,reine Erzählung‘ alles andere als der Regelfall sind. 
Historisch gesehen gibt es in der englischen (sowie europäischen) Literatur zahlreiche Beispiele für frühneuzeitliche Ekphrasen (vgl. Klarer 2001), denn die Dichterinnen und Dichter lagen mit den Maler/innen in einem Wettstreit darüber, wer die besseren, mithin lebendigeren und farbigeren Bilder liefern kann. Meist werden fiktive Kunstwerke beschrieben, etwa in Edmund Spensers Versepos The Faerie Queene (1590; vgl. zum Beispiel Bücher I-III) und Philip Sidneys Prosaromanze The New Arcadia (1583-84). Auch in der Literatur des 18. und 19. Jahrhunderts stehen Ekphrasen noch weitgehend im Dienste der enargeia (,Anschaulichkeit'). Jedoch setzt in der Moderne eine Problematisierung der von antiken Rhetoren gepriesenen Beschreibungskunst und der Techniken der Veranschaulichung und des ,Vor-Augen-Stellens“ (evidentia; hypotyposis) ein. In der modernen und postmodernen Literatur, die einer Poetik der Visualisierung folgen, lässt sich häufig ein Spannungsverhältnis zwischen eleutheromanie (,Beschreibungssucht') und der gleichzeitigen Subversion von Mimesis-Doktrin und Anschaulichkeitsrhetorik feststellen. Die hochkomplexe intermedial verfahrende angloamerikanische Literatur des 20. und 21. Jahrhunderts reflektiert notwendig die eigene Materialität. Die Auseinandersetzung mit dem Bild ist jedoch keinesfalls nur eine Defensivstrategie, sondern birgt Chancen, die der Literatur auch neue ästhetische Möglichkeiten und Dimensionen eröffnet, was sich an Charles Simics Gedichtband Dime-Store Alchemy. The Art of Joseph Cornell (1992) ebenso ablesen lässt wie an John Updikes Roman Seek My Face (2004), wo von Ekphrasen starke narrative und imaginative Impulse ausgehen. Die kanadische Gegenwartsautorin Margaret Atwood hat sich bereits in einem ihrer frühen Gedichte „This Is a Photograph of Me“ (1966) auf subversive Weise mit dem Thema Bildbeschreibung auseinander gesetzt:

This Is a Photograph of Me

It was taken some time ago.

At first it seems to be

a smeared

print: blurred lines and grey flecks

blended with the paper;

then, as you scan

it, you see in the left-hand corner

a thing that is like a branch: part of a tree

(balsam or spruce) emerging

and, to the right, halfway up

what ought to be a gentle

slope, a small frame house. 


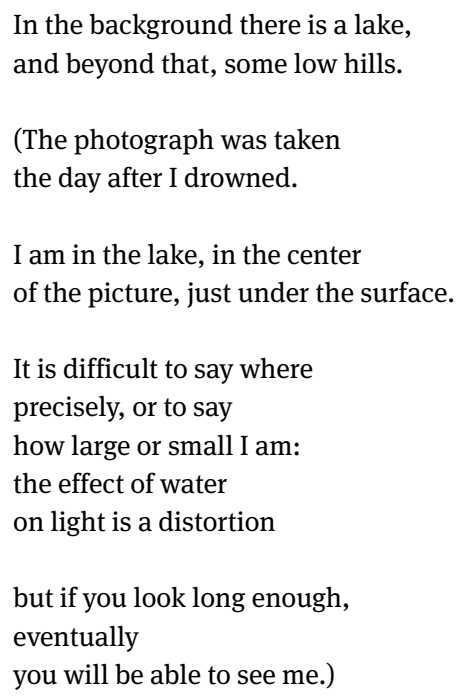

(Atwood 1985 [1966], 2292-2293)

In der ersten Gedichthälfte (Zeile 1-14) wird ein etwas unscharfes, vielleicht schon älteres Foto beschrieben. Die sachlich-präzise Beschreibung, die das Bild von links nach rechts nachzeichnet, zeigt ein Häuschen an einem kleinen See vor einem Hügelzug, porträtiert also ein typisch ländliches kanadisches Setting. Während man zunächst meint, es hier mit einer ekphrastischen Fotobeschreibung zu tun zu haben, wird in der zweiten, mit Klammern gerahmten Gedichthälfte schnell deutlich, dass es sich um einen Atwood-Text handelt, der wie immer an der Grenze zum Grotesken, zum gothic angesiedelt ist und viel schwarzen Humor besitzt. Es spricht nun ein lyrisches Ich, das bereits verstorben ist und sich somit als Tote aus einer unmöglichen narrativen Position heraus mit einer eigentlich unmöglichen Ekphrase an die Nachwelt wendet. Die Sprecherin ist im Foto nicht zu sehen, da die Leiche unter der Wasseroberfläche des Sees treibt und deshalb nicht genau geortet werden kann. Die Leserinnen und Leser werden eingeladen, länger ,hinzuschauen', ihre Aufmerksamkeit wird gedehnt, um das Sprecher-Ich doch noch zu entdecken.

Dass Atwood in ihrem ekphrastischen Gedicht anhand einer Fotografie - und nicht einer Zeichnung oder einem Gemälde - Mediengrenzen auslotet, überrascht nicht, wenn man sich an Susan Sontags berühmte Definition der Fotografie erinnert: „Jede Fotografie ist eine Art memento mori“ (Sontag 2000 [1977], 21). Bei Atwood handelt es sich um ein memento mori, das über Negativität funktioniert; gezeigt wird nämlich nicht eine Person, sondern niemand. Atwood setzt sich hier auf spielerische Weise mit dem indexikalischen Wert des visuellen Mediums 
Fotografie auseinander, indem sie diese wichtige Funktion der Fotografie infrage stellt. Ihre Beschreibung macht darüber hinaus deutlich, dass Bilder ohne Beschreibung und ,Beschriftung' keine spezifische zeitlich-räumliche Verortung und keine spezifische Botschaft haben. In diesem Sinne hatte schon Roland Barthes in seiner Rhetorik des Bildes (Barthes 1990 [1964]) erklärt, dass die Polysemie des Bildes durch die Sprache gezähmt wird (vgl. 1 BENTHIEN/WEINGART). Atwoods literarisches Spiel mit der Fotografie und ihrer Dokumentations- und Authentifizierungsfunktion eröffnet ihr die Möglichkeit, das Medium Fotografie im Medium Literatur kritisch zu beleuchten und die imaginäre Kraft von Literatur herauszustellen.

\section{Ausblick: Intermedialität im digitalen Zeitalter}

Seit einigen Jahrzehnten wird von Kulturtheoretikern eine rasant fortschreitende Marginalisierung von Sprache, Schrift und Druckkultur diagnostiziert und das Ende des gedruckten Buches heraufbeschworen (vgl. Mitchell 1994; Bolter 1996). Tatsächlich haben Zahl und Macht der Bilder im digitalen Zeitalter mit den immer kürzer werdenden Distributionszeiten, ihrem universellen Verbreitungsgrad und ihrer Manipulierbarkeit deutlich zugenommen. Fragen, die das intermediale Verhältnis von Texten und Bildern betreffen, sind in der Folge noch dringlicher geworden, gerade auch hinsichtlich der neuen Sorte hypertextuell kodierter fiktionaler Texte, die ausschließlich in einer elektronisch medialen Form existieren (vgl. Jannidis 1998; Simanowski 2002; Heibach 2003; Segeberg und Winko 2005) und Text, Bild und Diagramm intermedial verschränken. Michael Joyces Afternoon. A Story (1990), Stuart Moulthrops Victory Garden (1992), Simon Biggs' Great Wall of China (1996) und Douglas Adams' Starship Titanic (1997), eine novelization eines Computerspiels gleichen Namens von Terry Jones, gehörten zu den Wegbereitern der digitalen Literatur, der sogenannten hyperfiction. Computer-generierte hyperfiction hat nicht dieselbe Stabilität und lineare Form wie traditionelle Texte und stellt aufgrund ihrer interaktiven und multimedialen Form das Konzept einer rein monomedialen verbalen Kunst infrage. Denn stärker noch als gedruckte literarische Texte machen ihre digitalen Verwandten deutlich, was eigentlich für alle Texte, auch die gedruckten, gilt: Sie sind auf vielfältige Weise mit visuellen Elementen verknüpft.

Dass in der Gegenwartskunst und -kultur Mediengrenzen nicht nur nachdrücklich problematisiert, sondern auch offensichtlich durchlässig werden, hat auch Auswirkungen auf andere der hier diskutierten Konzepte, wie etwa die Ekphrasis. Wenn das konstante Recycling von Bildern und Geschichten und 
damit Phänomene wie Remediation (vgl. Bolter und Grusin 1999), Transmedialität (vgl. Wolf 2005 und 2011), Hybridisierung (vgl. Rajewsky 2005), Adaptation (vgl. Hutcheon 2006), Medienkonvergenz und transmedia story telling (das heißt die gleichzeitige Präsentation von Geschichten auf verschiedenen Medienplattformen; vgl. Jenkins 2006), eine so wichtige Rolle spielen wie heute, dann wird es immer schwieriger, bestimmten Bildern und Narrativen den Status von ,Originalen' zuzuschreiben. Zugespitzt formuliert kann man sagen, dass heute alles zu Ekphrasis geworden und in den nicht endenden Zyklus von Repräsentation von Repräsentation von Repräsentation und so weiter eingetreten ist. Diese Überlegung zur heutigen Rolle von Ekphrasis verdeutlicht, dass die neuen digitalen Medien neue inter-/mediale Möglichkeiten eröffnet haben, die nach einer Erweiterung älterer Theoretisierungsansätze und Konzeptionalisierungen intermedialer Phänomene und kultureller Praktiken verlangen, um das Potential und die Funktionen intermedialer Konstellationen und Kunstformen austarieren und beschreiben zu können.

\section{Literaturverzeichnis}

Adler, Jeremy, und Ulrich Ernst. Text als Figur. Weinheim: Acta Humaniora, VCH, 1987.

Atwood, Margaret. „This Is a Photograph of Me“ [1966]. The Norton Anthology of Literature by Women. Hrsg. von Sandra M. Gilbert und Susan Gubar. New York, NY: Norton, 1985. 2292-2293.

Barthes, Roland. „Rhetorik des Bildes“ [1964]. Der entgegenkommende und der stumpfe Sinn. Kritische Essays III. Übers. von Dieter Horning. Frankfurt am Main: Suhrkamp, 1990. 28-46.

Bender, John B. Spenser and Literary Pictorialism. Princeton, NJ: Princeton University Press, 1972.

Biggs, Simon. Great Wall of China. http://www.littlepig.org.uk/wall/greatwall.htm. 1996 (12. Mai 2014).

Boehm, Gottfried, und Helmut Pfotenhauer (Hrsg.). Beschreibungskunst - Kunstbeschreibung. Ekphrasis von der Antike bis zur Gegenwart. München: Fink, 1995.

Bolter, Jay David. „Ekphrasis, Virtual Reality, and the Future of Writing“. The Future of the Book. Hrsg. von Geoffrey Nunberg. Berkeley, CA: University of California Press, 1996. 253-272.

Bolter, Jay David, und Richard Grusin. Remediation: Understanding New Media. Cambridge, MA: MIT Press, 1999.

Dickens, Charles. Oliver Twist. London: Penguin, 1971 [1838].

Faulstich, Werner. Medientheorien. Göttingen: Vandenhoeck \& Ruprecht, 1991.

Foer, Jonathan Safran. Extremely Loud and Incredibly Close. London: Hamish Hamilton, Penguin, 2005.

Gardner, Helen (Hrsg). The Metaphysical Poets. Harmondsworth: Penguin, 1957.

Georgi, Claudia. Liveness on Stage. Intermedial Challenges in Contemporary British Theatre and Performance. Berlin und Boston, MA: de Gruyter, 2014. 
Hagstrum, Jean H. The Sister Arts. The Tradition of Literary Pictorialism and English Poetry from Dryden to Gray. Chicago, IL: The University of Chicago Press, 1958.

Hansen-Löve, Aage A. „Intermedialität und Intertextualität. Probleme der Korrelation von Wort- und Bildkunst. Am Beispiel der russischen Moderne“. Dialog der Texte. Hamburger Kolloquium zur Intertextualität. Hrsg. von Wolf Schmid und Wolf-Dieter Stempel. Wien: Gesellschaft zur Förderung slawistischer Studien, 1983. 291-360.

Heffernan, James A. W. „Ekphrasis and Representation“. New Literary History 22 (1991): 297-316.

Heffernan, James A. W. Museum of Words. The Poetics of Ekphrasis from Homer to Ashbery. Chicago, IL, und London: The University of Chicago Press, 1993.

Heibach, Christiane. Literatur im elektronischen Raum. Frankfurt am Main: Suhrkamp, 2003. Henkel, Arthur, und Albrecht Schöne (Hrsg.). Emblemata. Handbuch zur Sinnbildkunst des XVI. und XVII. Jahrhunderts. Stuttgart und Weimar: Metzler, 1996 [1967].

Higgins, Dick. Horizons. The Poetics and Theory of the Intermedia (1966). Carbondale, IL, und Edwardsville, IL: Southern Illinois University Press, 1984.

Hollander, John. The Gazer's Spirit. Poems Speaking to Silent Works of Art. Chicago, IL, und London: The University of Chicago Press, 1995.

Horaz [Quintus Horatius Flaccus]. „De arte poetica liber/Das Buch von der Dichtkunst“ [14 v. Chr.]. Sämtliche Werke. Lateinisch und Deutsch. Teil I nach Kayser, Nordenflycht, Burger hrsg. von Hans Färber, Teil II übers. und bearb. von Hans Färber und Wilhelm Schöne. Darmstadt: Wissenschaftliche Buchgesellschaft, 1967. 230-259.

Hutcheon, Linda. A Theory of Adaptation. New York, NY: Routledge, 2006.

Jannidis, Fotis. „Computerphilologie“. Metzler Lexikon Literatur- und Kulturtheorie. Ansätze Personen - Grundbegriffe. Hrsg. von Ansgar Nünning. Stuttgart und Weimar: Metzler, 1998. 70-72.

Jenkins, Henry. Convergence Cultures. Where Old and New Media Collide. New York, NY, und London: New York University Press, 2006.

Klarer, Mario. „Introduction“. Word \& Image 15.1 (1999): 1-4.

Klarer, Mario. Ekphrasis. Bildbeschreibung als Repräsentationstheorie bei Spenser, Sidney, Lyly und Shakespeare. Tübingen: Niemeyer, 2001.

Krieger, Murray. Ekphrasis. The Illusion of the Natural Sign. Baltimore, MD: The Johns Hopkins University Press, 1992.

Langer, Susanne K. Philosophie aufneuem Wege. Das Symbol im Denken, im Ritus und in der Kunst. Frankfurt am Main: Fischer, 1984 [1942].

Lessing, Gotthold Ephraim. Laokoon oder Über die Grenzen der Malerei und Poesie. Stuttgart: Reclam, 1994 [1766].

Mitchell, W. J. T. „Was ist ein Bild?“ Bildlichkeit. Hrsg. von Volker Bohn. Frankfurt am Main: Suhrkamp, 1990. 17-68.

Mitchell, W. J. T. Picture Theory. Essays on Verbal and Visual Representation, Chicago, IL, und London: The University of Chicago Press, 1994.

Mosher, Harold F., Jr. „Towards a Poetics of Descriptized Narration“. Poetics Today 3 (1991): 425-445.

Paech, Joachim, und Jens Schröter (Hrsg.). Intermedialität - Analog/Digital. Theorien, Methoden, Analysen. München: Fink, 2008.

Preimesberger, Rudolf. Paragons and Paragone. Van Eyck, Raphael, Michelangelo, Caravaggio and Bernini. Los Angeles, CA: Getty Research Institute, 2011.

Rajewsky, Irina O. Intermedialität. Tübingen und Basel: Francke, 2002. 
Rajewsky, Irina O. „Intermedialität - Eine Begriffsbestimmung“. Intermedialität im Deutschunterricht. Hrsg. von Marion Bönnighausen und Heidi Rösch. Baltmannsweiler: Schneider Verlag Hohengehren, 2004. 8-30.

Rajewsky, Irina 0. „Intermediality, Intertextuality, and Remediation: A Literary Perspective on Intermediality“. Intermédialités 6 (2005): 43-64.

Rajewsky, Irina 0. „Border Talks: The Problematic Status of Media Borders in the Current Debate about Intermediality“. Media Borders, Multimodality and Intermediality. Hrsg. von Lars Elleström. Basingstoke: Palgrave Macmillan, 2010. 51-68.

Redniss, Lauren. Radioactive - Marie \& Pierre Curie. A Tale of Love and Fallout. New York, NY: Harper Collins, 2010.

Rippl, Gabriele. Beschreibungs-Kunst. Zur intermedialen Poetik angloamerikanischer Ikontexte (1880-2000). München: Fink, 2005a.

Rippl, Gabriele. „Literatur und (visuelle) Medien in der frühen Neuzeit“. Kulturgeschichte der englischen Literatur. Von der Renaissance bis zur Gegenwart. Hrsg. von Vera Nünning. Tübingen und Basel: Francke, utb, 2005b. 36-47.

Rippl, Gabriele. „Intermediale Poetik: Ekphrasis und der ,iconic turn“ in der Literatur/wissenschaft“. Bilder: ein (neues) Leitmedium? Hrsg. von Torsten Hoffmann und Gabriele Rippl. Göttingen: Wallstein, 2006. 93-107.

Rippl, Gabriele. „Film and Media Studies“. English and American Studies. Theory and Practice. Hrsg. von Martin Middeke, Timo Müller, Christina Wald und Hubert Zapf. Stuttgart: Metzler, 2012. 314-332.

Robillard, Valerie, und Els Jongeneel (Hrsg.). Pictures into Words. Theoretical and Descriptive Approaches to Ekphrasis. Amsterdam: VU University Press, 1998.

Ronen, Ruth. „Description, Narrative and Representation“. Narrative 5.3 (1997): 274-286.

Ryan, Marie-Laure. „Introduction“. Narrative Across Media. The Languages of Storytelling. Hrsg. von Marie-Laure Ryan. Lincoln, NE, i. a.: University of Nebraska Press, 2004. 1-40.

Ryan, Marie-Laure. „On the Theoretical Foundations of Transmedial Narratology“. Narratology beyond Criticism. Mediality, Disciplinarity. Hrsg. von Jan Christoph Meister. Berlin: De Gruyter, 2005. 1-23.

Schmitz-Emans, Monika. Die Literatur, die Bilder und das Unsichtbare. Spielformen literarischer Bildinterpretation vom 18. bis zum 20. Jahrhundert. Würzburg: Königshausen \& Neumann, 1999.

Segeberg, Harro, und Simone Winko (Hrsg.). Digitalität und Literatur. Zur Zukunft der Literatur. München: Fink, 2005.

Simanowski, Roberto. Interfictions. Vom Schreiben im Netz. Frankfurt am Main: Suhrkamp, 2002.

Sontag, Susan. Über Fotografie. Essays. Übers. von Mark W. Rien. 12. Aufl. Frankfurt am Main: Fischer, 2000 [1977].

Todorow, Almut. „Intermedialität“. Historisches Wörterbuch der Rhetorik Bd. 10. Hrsg. von Gert Ueding. Tübingen: Max Niemeyer, 2011. 299-410.

Torgovnick, Marianna. The Visual Arts, Pictorialism, and the Novel. James, Lawrence, and Woolf. Princeton, NJ: Princeton University Press, 1985.

Voßkamp, Wilhelm, und Brigitte Weingart. „Sichtbares und Sagbares. Text-Bild-Verhältnisse Einleitung“. Sichtbares und Sagbares. Text-Bild-Verhältnisse. Hrsg. von Wilhelm Voßkamp und Brigitte Weingart. Köln: DuMont 2005. 7-22.

Wagner, Peter (Hrsg.). Icons - Texts - Iconotexts. Essays on Ekphrasis and Intermediality, Berlin: De Gruyter, 1996. 
Wandhoff, Haiko. Ekphrasis. Kunstbeschreibungen und virtuelle Räume in der Literatur des Mittelalters. Berlin und New York, NY: De Gruyter, 2003.

Weingart, Brigitte. „Where is your rupture? Zum Transfer zwischen Text- und Bildtheorie“. Die Adresse des Mediums. Hrsg. von Stefan Andriopoulos, Gabriele Schabacher und Eckhard Schumacher. Köln: DuMont, 2001. 136-157.

Weingart, Brigitte. „Bastards: Text/Image Hybrids in Pop Writing by Rolf Dieter Brinkmann and Others". Media, Culture, and Mediality. New Insights into the Current State of Research. Hrsg. von Ludwig Jäger, Erika Linz und Irmela Schneider. Bielefeld: transcript, 2010. 429-461.

Weisstein, Ulrich (Hrsg.). Literatur und Bildende Kunst. Ein Handbuch zur Theorie und Praxis eines komparatistischen Grenzgebietes. Berlin: Schmidt, 1992.

Williams, William Carlos. „XXII (The Red Wheelbarrow)“ [1923]. The Collected Poems of William Carlos Williams Bd. 2: 1909-1939. Hrsg. von A. Walton Litz und Christopher MacGowan. New York, NY: New Directions, 1991. 224.

Wolf, Werner. „Das Problem der Narrativität in Literatur, bildender Kunst und Musik. Ein Beitrag zu einer intermedialen Erzähltheorie“. Erzähltheorie transgenerisch, intermedial, interdisziplinär. Hrsg. von Vera Nünning und Ansgar Nünning. Trier: Wissenschaftlicher Verlag Trier, 2002. 23-104.

Wolf, Werner. „Intermediality“. Routledge Encyclopedia of Narrative Theory. Hrsg. von David Herman, Manfred Jahn und Marie-Laure Ryan. London und New York, NY: Routledge, 2005. 252-256.

Wolf, Werner. „Narratology and Media(lity): The Transmedial Expansion of a Literary Discipline and Possible Consequences“. Current Trends in Narratology. Hrsg. von Greta Olson. Berlin und New York, NY: De Gruyter, 2011. 146-180.

Yacobi, Tamar. „Pictorial Models and Narrative Ekphrasis“. Poetics Today 16.4 (1995): 599-649. 